\title{
ADAPTIVESYNCHRONIZER DESIGN FOR THE HYBRID SYNCHRONIZATION OF HYPERCHAOTIC ZHENGAND HYPERCHAOTIC YU SYSTEMS
}

\author{
Sundarapandian Vaidyanathan \\ Research and Development Centre, Vel Tech Dr. RR \& Dr. SR Technical University \\ Avadi, Chennai-600 062, Tamil Nadu, INDIA \\ sundarvtuegmail.com
}

\begin{abstract}
This paper derives new adaptive synchronizers for the hybrid synchronization of hyperchaotic Zheng systems (2010) and hyperchaotic Yu systems (2012). In the hybrid synchronization design of master and slave systems, one part of the systems, viz. their odd states, are completely synchronized (CS), while the other part, viz. their even states, are completely anti-synchronized (AS) so that CS and AS co-exist in the process of synchronization. The research problem gets even more complicated, when the parameters of the hyperchaotic systems are not known and we handle this complicate problem using adaptive control. The main results of this research work are established via adaptive control theory andLyapunov stability theory. MATLAB plotsusing classical fourth-order Runge-Kutta method have been depictedfor the new adaptive hybrid synchronization results for the hyperchaotic Zheng and hyperchaotic Yu systems.
\end{abstract}

\section{KEYWORDS}

Hybrid Synchronization, Adaptive Control, Chaos, Hyperchaos, Hyperchaotic Systems.

\section{INTRODUCTION}

Since thediscovery by the German scientist,O.E.Rössler ([1], 1979), hyperchaotic systems have found many applicationsin areas like neural networks [2],oscillators [3], communication [4-5], encryption [6], synchronization [7], etc. In chaos theory, hyperchaotic system is usually defined as a chaotic system having two or more positive Lyapunov exponents. Hyperchaotic systems have many attractive features like high efficiency, high capacity, high security, etc.

For the synchronization of chaotic systems, there are many methods available in the chaos literature like OGY method [8], PC method [9],backstepping method [10-12], sliding control method [13-15], active control method [16-17], adaptive control method [18-19], sampled-data feedback control [20], time-delay feedback method [21], etc.

In the hybrid synchronization of a pair of chaotic systems called the master and slave systems, one part of the systems, viz. the odd states, are completely synchronized (CS), while the other part of the systems, viz. the even states, are anti-synchronized so that CS and AS co-exist in the process of synchronization of the two systems.

This paper focuses upon adaptive controller design for the hybrid synchronization of hyperchaotic Zheng systems ([22], 2010) and hyperchaotic Yusystems ([23], 2012) with unknown parameters. 
The main results derived in this paper have been proved using adaptive control theory [24] andLyapunov stability theory [25]..

\section{Adaptive Control Methodologyfor Hybrid Synchronization}

The master system is described by the chaotic dynamics

$$
\dot{x}=A x+f(x)
$$

where $A$ is the $n \times n$ matrix of the system parameters and $f: R^{n} \rightarrow R^{n}$ is the nonlinear part. The slave system is described by the chaotic dynamics

$$
\dot{y}=B y+g(y)+u
$$

where $B$ is the $n \times n$ matrix of the system parameters and $g: R^{n} \rightarrow R^{n}$ is the nonlinear part For the pair of chaotic systems (1) and (2), the hybrid synchronization erroris defined as

$$
e_{i}=\left\{\begin{array}{cc}
y_{i}-x_{i}, & \text { if } i \text { is odd } \\
y_{i}+x_{i}, & \text { if } i \text { is even }
\end{array}\right.
$$

The error dynamics is obtained as

$$
\dot{e}_{i}= \begin{cases}\sum_{j=1}^{n}\left(b_{i j} y_{j}-a_{i j} x_{j}\right)+g_{i}(y)-f_{i}(x)+u_{i} & \text { if } i \text { is odd } \\ \sum_{j=1}^{n}\left(b_{i j} y_{j}+a_{i j} x_{j}\right)+g_{i}(y)+f_{i}(x)+u_{i} & \text { if } i \text { is even }\end{cases}
$$

The design goal is to find a feedback controller $u$ so that

$$
\lim _{t \rightarrow \infty}\|e(t)\|=0 \text { for all } e(0) \in R^{n}
$$

Using the matrix method, we consider a candidate Lyapunov function

$V(e)=e^{T} P e$,

where $P$ is a positive definite matrix. It is noted that $V: R^{n} \rightarrow R$ is a positive definite function.

If we find a feedback controller $u$ so that

$\dot{V}(e)=-e^{T} Q e$,

where $Q$ is a positive definite matrix, then $\dot{V}: R^{n} \rightarrow R$ is a negative definite function.

Thus, by Lyapunov stability theory [25], the error dynamics (4) is globally exponentially stable. Hence, the states of the chaotic systems (1) and (2) will be globally and exponentially hybrid synchronized for all initial conditions $x(0), y(0) \in R^{n}$. When the system parameters are unknown, we use estimates for them and find a parameter update law using Lyapunov approach. 


\section{4-D HYPERCHAOTIC SYSTEMS}

The 4-D hyperchaotic Zhengsystem ([22], 2010) has the dynamics

$$
\begin{aligned}
& \dot{x}_{1}=a\left(x_{2}-x_{1}\right)+x_{4} \\
& \dot{x}_{2}=b x_{1}+c x_{2}+x_{1} x_{3}+x_{4} \\
& \dot{x}_{3}=-x_{1}^{2}-r x_{3} \\
& \dot{x}_{4}=-d x_{2}
\end{aligned}
$$

where $a, b, c, r, d$ are constant, positive parameters of the system.

The 4-D Zheng system (8) exhibits a hyperchaotic attractor for the parametric values

$$
a=20, \quad b=14, c=10.6, \quad d=4, \quad r=2.8
$$

The Lyapunov exponents of the system (8) for the parametric values in (9) are

$$
L_{1}=1.8892, \quad L_{2}=0.2268, \quad L_{3}=0, \quad L_{4}=-14.4130
$$

Since there are two positive Lyapunov exponents in (10), the Zheng system (8) is hyperchaotic for the parametric values (9).

The strange attractor of the hyperchaotic Zheng system is displayed in Figure 1.

The 4-D hyperchaotic Yu system ([23], 2012) has the dynamics

$$
\begin{aligned}
& \dot{x}_{1}=\alpha\left(x_{2}-x_{1}\right) \\
& \dot{x}_{2}=\beta x_{1}-x_{1} x_{3}+\gamma x_{2}+x_{4} \\
& \dot{x}_{3}=e^{x_{1} x_{2}}-\delta x_{3} \\
& \dot{x}_{4}=-\varepsilon x_{1}
\end{aligned}
$$

where $\alpha, \beta, \gamma, \delta, \varepsilon$ are constant, positive parameters of the system.

The 4-D Yu system (11) exhibits a hyperchaotic attractor for the parametric values

$$
\alpha=10, \beta=40, \gamma=1, \delta=3, \varepsilon=8
$$

The Lyapunov exponents of the system (11) for the parametric values in (12) are

$$
L_{1}=1.6877, \quad L_{2}=0.1214, \quad L_{3}=0, \quad L_{4}=-13.7271
$$

Since there are two positive Lyapunov exponents in (13), theYusystem (11) is hyperchaotic for the parametric values (12).

The strange attractor of the hyperchaotic Yu system is displayed in Figure 2. 
International Journal of Information Technology Convergence and Services (IJITCS) Vol.3, No.2, April 2013
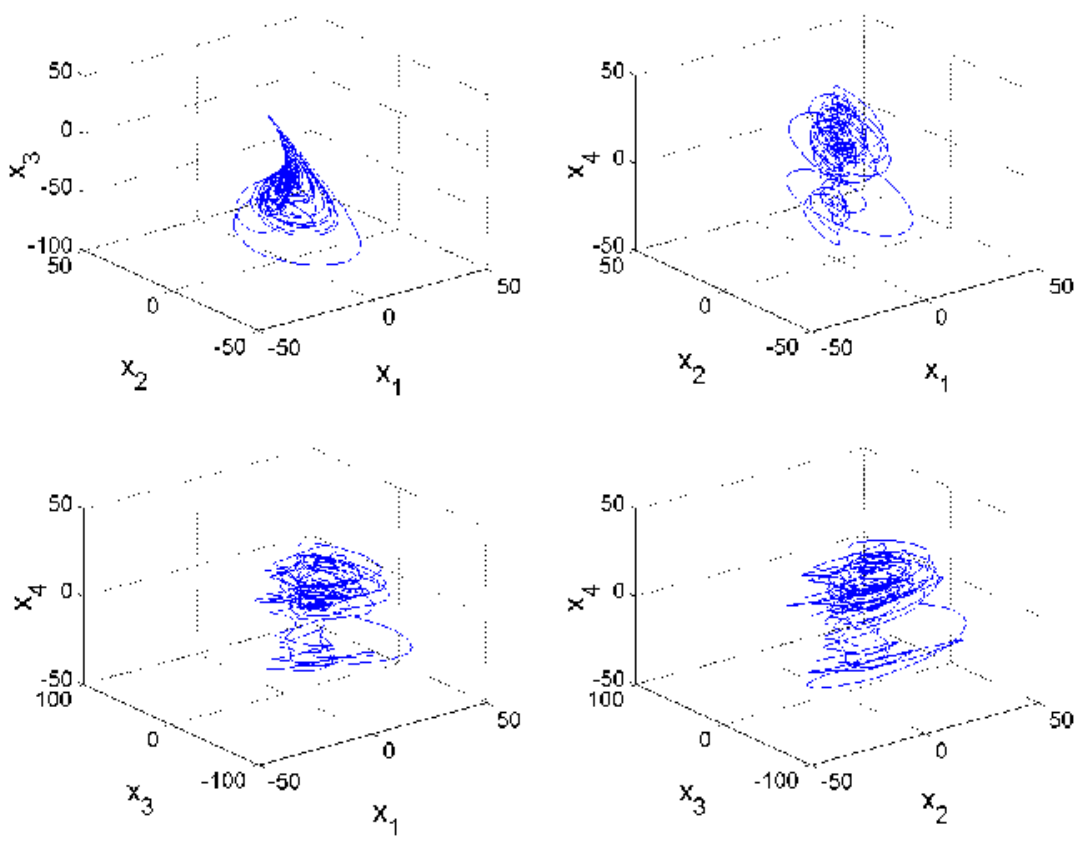

Figure 1. The State Portrait of the HyperchaoticZhengSystem
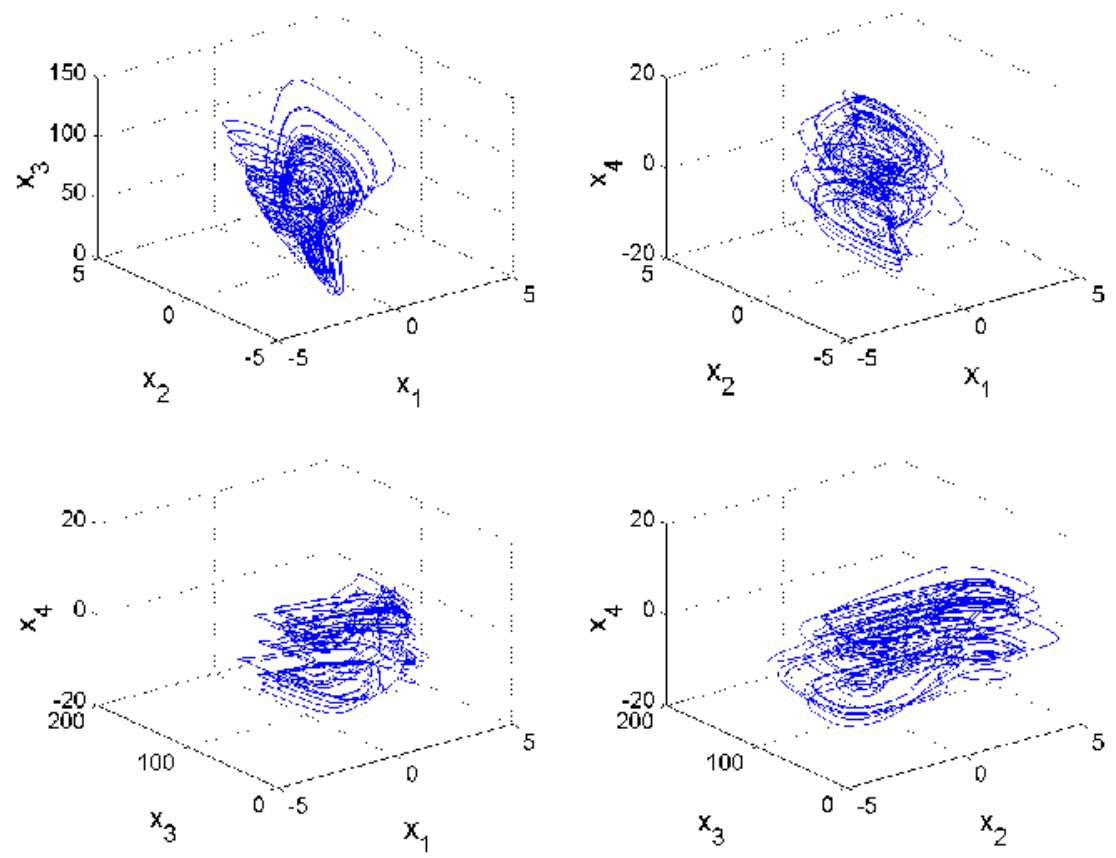

Figure 2. The State Portrait of the Hyperchaotic Yu System 
International Journal of Information Technology Convergence and Services (IJITCS) Vol.3, No.2, April 2013

\section{AdaptiveControl Design for the HybridSynchronization of HYPERCHAOTIC ZHENG SYSTEMS}

In this section, we design an adaptivesynchronizer for the hybrid synchronization of two identical hyperchaotic Zheng systems (2010) with unknown parameters.

The hyperchaotic Zheng system is taken as the master system, whose dynamics isgiven by

$$
\begin{aligned}
& \dot{x}_{1}=a\left(x_{2}-x_{1}\right)+x_{4} \\
& \dot{x}_{2}=b x_{1}+c x_{2}+x_{1} x_{3}+x_{4} \\
& \dot{x}_{3}=-x_{1}^{2}-r x_{3} \\
& \dot{x}_{4}=-d x_{2}
\end{aligned}
$$

where $a, b, c, d, r$ are unknown parameters of the system and $x \in R^{4}$ is the state of the system.

The hyperchaotic Zheng system is also taken as the slave system, whose dynamics is given by

$$
\begin{aligned}
& \dot{y}_{1}=a\left(y_{2}-y_{1}\right)+y_{4}+u_{1} \\
& \dot{y}_{2}=b y_{1}+c y_{2}+y_{1} y_{3}+y_{4}+u_{2} \\
& \dot{y}_{3}=-y_{1}^{2}-r y_{3}+u_{3} \\
& \dot{y}_{4}=-d y_{2}+u_{4}
\end{aligned}
$$

where $y \in R^{4}$ is the state and $u_{1}, u_{2}, u_{3}, u_{4}$ are the adaptivecontrollers to be designed using estimates $\hat{a}(t), \hat{b}(t), \hat{c}(t), \hat{d}(t), \hat{r}(t)$ of the unknown parameters $a, b, c, d, r$, respectively.

For the hybrid synchronization, the error $e$ is defined as

$$
e_{1}=y_{1}-x_{1}, e_{2}=y_{2}+x_{2}, e_{3}=y_{3}-x_{3}, e_{4}=y_{4}+x_{4}
$$

A simple calculation gives the error dynamics

$$
\begin{aligned}
& \dot{e}_{1}=a\left(y_{2}-x_{2}-e_{1}\right)+y_{4}-x_{4}+u_{1} \\
& \dot{e}_{2}=b\left(y_{1}+x_{1}\right)+c e_{2}+e_{4}+y_{1} y_{3}+x_{1} x_{3}+u_{2} \\
& \dot{e}_{3}=-r e_{3}-y_{1}^{2}+x_{1}^{2}+u_{3} \\
& \dot{e}_{4}=-d e_{2}+u_{4}
\end{aligned}
$$

Next, we choose a nonlinear controller for achieving hybrid synchronization as

$$
\begin{aligned}
& u_{1}=-\hat{a}(t)\left(y_{2}-x_{2}-e_{1}\right)-y_{4}+x_{4}-k_{1} e_{1} \\
& u_{2}=-\hat{b}(t)\left(y_{1}+x_{1}\right)-\hat{c}(t) e_{2}-e_{4}-y_{1} y_{3}-x_{1} x_{3}-k_{2} e_{2} \\
& u_{3}=\hat{r}(t) e_{3}+y_{1}^{2}-x_{1}^{2}-k_{3} e_{3} \\
& u_{4}=\hat{d}(t) e_{2}-k_{4} e_{4}
\end{aligned}
$$


In Eq. (18), $k_{i},(i=1,2,3,4)$ are positive gains and $\hat{a}(t), \hat{b}(t), \hat{c}(t), \hat{d}(t), \hat{r}(t)$ are estimates of the unknown parameters $a, b, c, d, r$, respectively.

By the substitution of (18) into (17), the error dynamics is determined as

$$
\begin{aligned}
& \dot{e}_{1}=(a-\hat{a}(t))\left(y_{2}-x_{2}-e_{1}\right)-k_{1} e_{1} \\
& \dot{e}_{2}=(b-\hat{b}(t))\left(y_{1}+x_{1}\right)+(c-\hat{c}(t)) e_{2}-k_{2} e_{2} \\
& \dot{e}_{3}=-(r-\hat{r}(t)) e_{3}-k_{3} e_{3} \\
& \dot{e}_{4}=-(d-\hat{d}(t)) e_{2}-k_{4} e_{4}
\end{aligned}
$$

Next, we define the parameter estimation errors as

$e_{a}(t)=a-\hat{a}(t), e_{b}(t)=b-\hat{b}(t), e_{c}(t)=c-\hat{c}(t), e_{d}(t)=d-\hat{d}(t), e_{r}(t)=r-\hat{r}(t)$

Differentiating (20) with respect to $t$, we get

$$
\dot{e}_{a}(t)=-\dot{\hat{a}}(t), \dot{e}_{b}(t)=-\dot{\hat{b}}(t), \dot{e}_{c}(t)=-\dot{\hat{c}}(t), \dot{e}_{d}(t)=-\dot{\hat{d}}(t), \quad \dot{e}_{r}(t)=-\dot{\hat{r}}(t)
$$

In view of (20), we can simplify the error dynamics (19) as

$$
\begin{aligned}
& \dot{e}_{1}=e_{a}\left(y_{2}-x_{2}-e_{1}\right)-k_{1} e_{1} \\
& \dot{e}_{2}=e_{b}\left(y_{1}+x_{1}\right)+e_{c} e_{2}-k_{2} e_{2} \\
& \dot{e}_{3}=-e_{r} e_{3}-k_{3} e_{3} \\
& \dot{e}_{4}=-e_{d} e_{2}-k_{4} e_{4}
\end{aligned}
$$

We take the quadratic Lyapunov function

$$
V=\frac{1}{2}\left(e_{1}^{2}+e_{2}^{2}+e_{3}^{2}+e_{4}^{2}+e_{a}^{2}+e_{b}^{2}+e_{c}^{2}+e_{d}^{2}+e_{r}^{2}\right),
$$

Which is a positive definite function on $R^{9}$.

When we differentiate (22) along the trajectories of (19) and (21), we get

$$
\begin{aligned}
\dot{V}= & -k_{1} e_{1}^{2}-k_{2} e_{2}^{2}-k_{3} e_{3}^{2}-k_{4} e_{4}^{2}+e_{a}\left[e_{1}\left(y_{2}-x_{2}-e_{1}\right)-\dot{\hat{a}}\right]+e_{b}\left[e_{2}\left(y_{1}+x_{1}\right)-\dot{\hat{b}}\right] \\
& +e_{c}\left[e_{2}^{2}-\dot{\hat{c}}\right]+e_{d}\left[-e_{2} e_{4}-\dot{\hat{d}}\right]+e_{r}\left[-e_{3}^{2}-\dot{\hat{r}}\right]
\end{aligned}
$$

In view of Eq. (24), we take the parameter update law as

$$
\begin{array}{lll}
\dot{\hat{a}}=e_{1}\left(y_{2}-x_{2}-e_{1}\right)+k_{5} e_{a}, & \dot{\hat{b}}=e_{2}\left(y_{1}+x_{1}\right)+k_{6} e_{b}, & \dot{\hat{c}}=e_{2}^{2}+k_{7} e_{c} \\
\dot{\hat{d}}=-e_{2} e_{4}+k_{8} e_{d}, & \dot{\hat{r}}=-e_{3}^{2}+k_{9} e_{r}
\end{array}
$$

Theorem 4.1 The adaptive control law (18) along with the parameter update law (25), where $k_{i},(i=1,2, \ldots, 9)$ are positive gains, achieves global and exponential hybrid synchronization of 
International Journal of Information Technology Convergence and Services (IJITCS) Vol.3, No.2, April 2013

the identical hyperchaotic Zheng systems (14) and (15), where $\hat{a}(t), \hat{b}(t), \hat{c}(t), \hat{d}(t), \hat{r}(t)$ are estimates of the unknown parameters $a, b, c, d, r$, respectively. In addition, the parameter estimation errors $e_{a}, e_{b}, e_{c}, e_{d}, e_{r}$ converge to zero exponentially for all initial conditions.

Proof.We prove the above result using Lyapunov stability theory [25].

Substituting the parameter update law (25) into (24), we get

$$
\dot{V}=-k_{1} e_{1}^{2}-k_{2} e_{2}^{2}-k_{3} e_{3}^{2}-k_{4} e_{4}^{2}-k_{5} e_{a}^{2}-k_{6} e_{b}^{2}-k_{7} e_{c}^{2}-k_{8} e_{d}^{2}-k_{9} e_{r}^{2}
$$

which is a negative definite function on $R^{9}$.

This shows that the hybrid synchronization errors $e_{1}(t), e_{2}(t), e_{3}(t), e_{4}(t)$ and the parameter estimation errors $e_{a}(t), e_{b}(t), e_{c}(t), e_{d}(t), e_{r}(t)$ are globally exponentially stable for all initial conditions. This completes the proof.

Next, we use MATLAB to demonstrate our hybrid synchronization results.

The classical fourthorder Runge-Kutta method with time-step $h=10^{-8}$ has been applied to solve the hyperchaotic Zheng systems (14) and (15) with the adaptive nonlinear controller(18) and the parameter update law (25). The feedback gains arechosen as $k_{i}=5,(i=1,2, \ldots, 9)$.

The parameters of the hyperchaotic Zheng systems are taken as in the hyperchaotic case, i.e.

$$
a=20, \quad b=14, \quad c=10.6, d=4, \quad r=2.8
$$

For simulations, the initial conditions of the hyperchaotic Zheng system (14) are chosen as

$$
x_{1}(0)=24, \quad x_{2}(0)=-15, \quad x_{3}(0)=-6, \quad x_{4}(0)=18
$$

Also, the initial conditions of the hyperchaotic Zheng system (15) are chosen as

$$
y_{1}(0)=12, \quad y_{2}(0)=-9, \quad y_{3}(0)=26, \quad y_{4}(0)=-6
$$

Also, the initial conditions of the parameter estimates are chosen as

$$
\hat{a}(0)=9, \hat{b}(0)=-7, \quad \hat{c}(0)=8, \hat{d}(0)=2, \hat{r}(0)=-5
$$

Figure 3 depicts the hybrid synchronization of the identical hyperchaoticZheng systems.

Figure 4 depicts the time-history of the hybrid synchronization errors $e_{1}, e_{2}, e_{3}, e_{4}$.

Figure 5 depicts the time-history of the parameter estimation errors $e_{a}, e_{b}, e_{c}, e_{d}, e_{r}$. 
International Journal of Information Technology Convergence and Services (IJITCS) Vol.3, No.2, April 2013
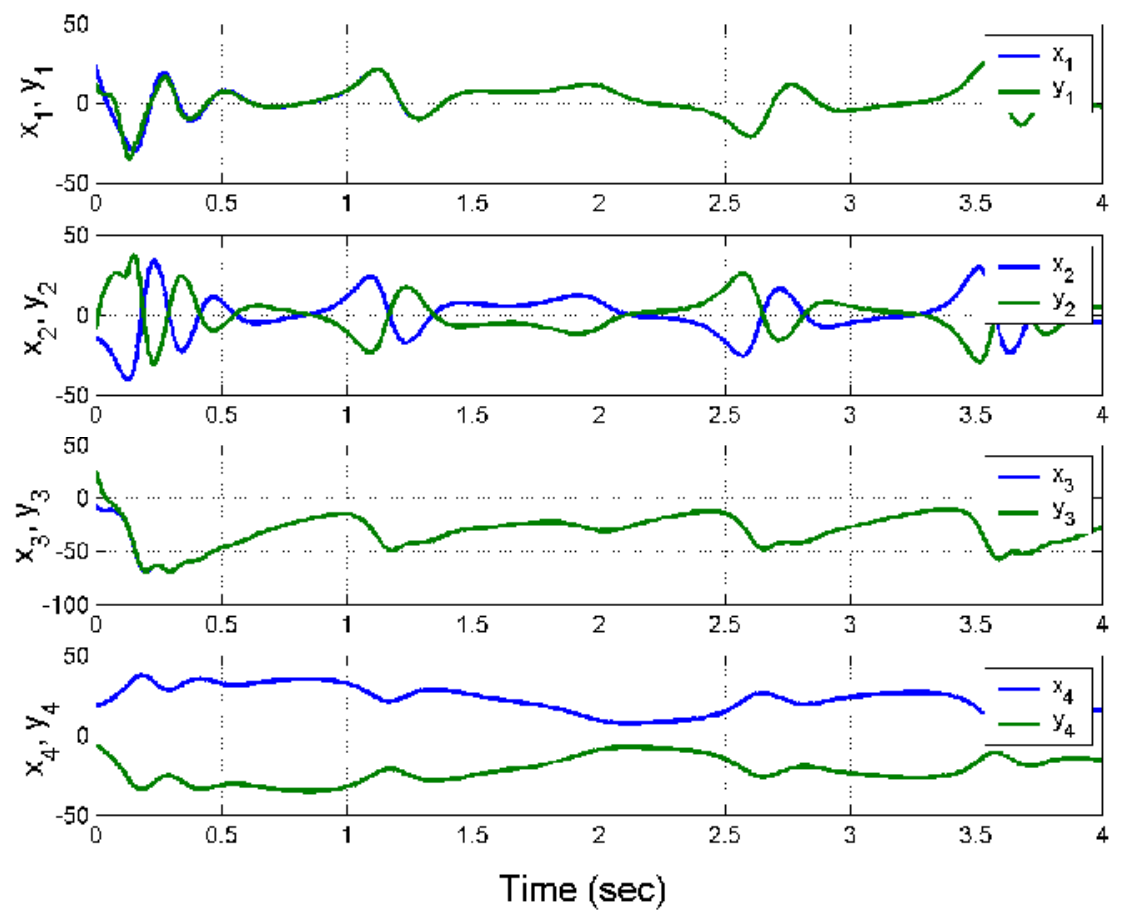

Figure 3.Hybrid Synchronization of Identical Hyperchaotic Zheng Systems

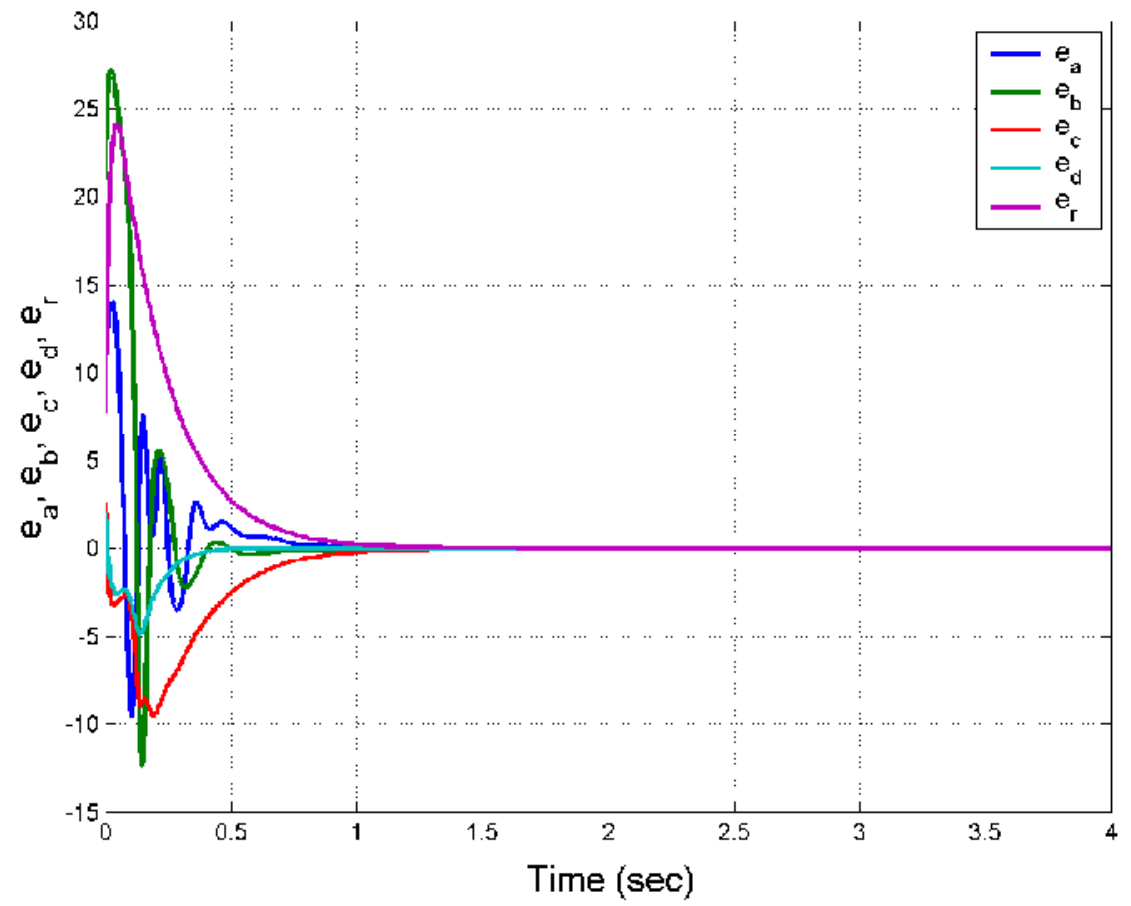

Figure 4. Time-History of the Hybrid Synchronization Errors $e_{1}, e_{2}, e_{3}, e_{4}$ 


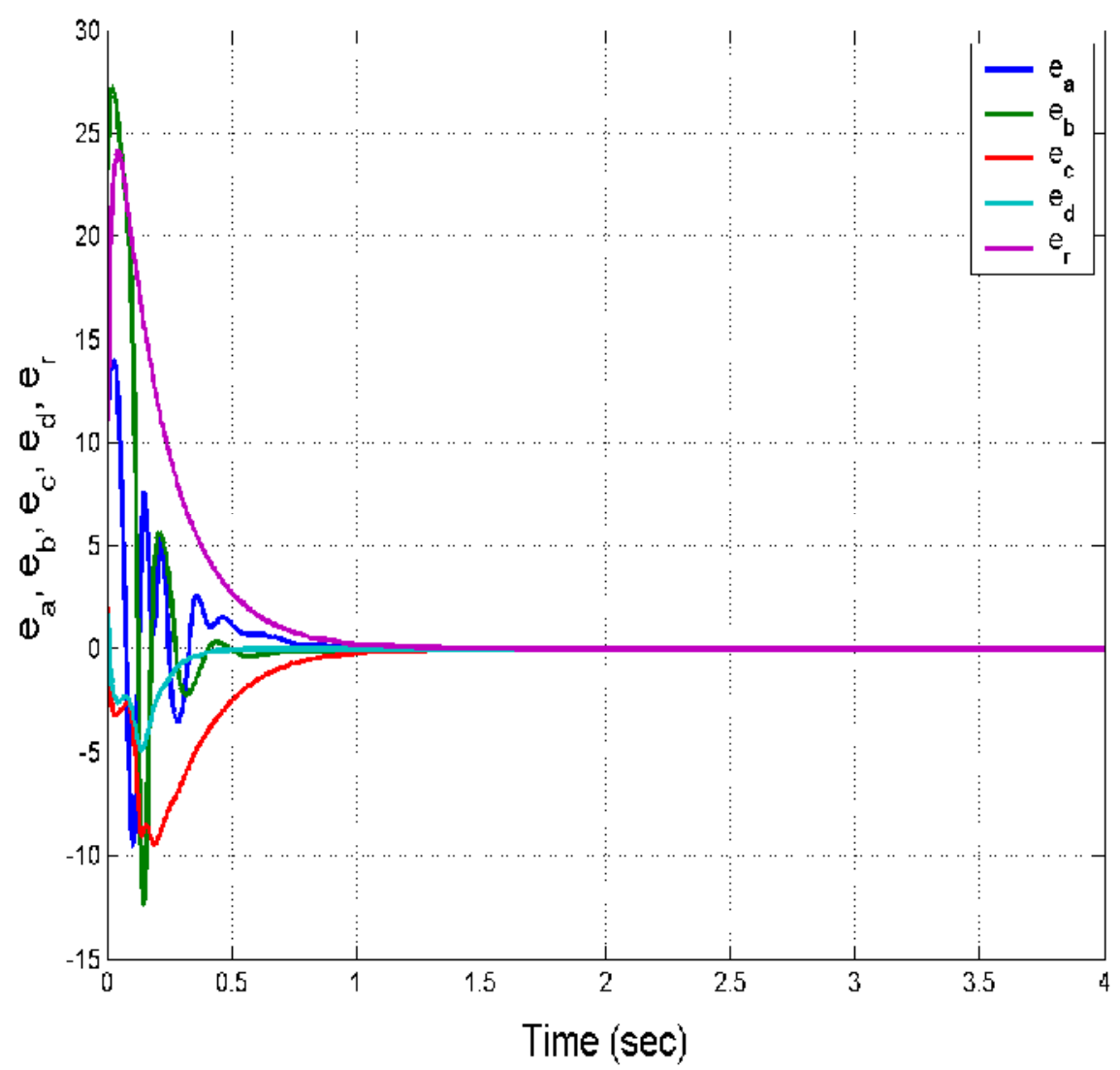

Figure 5. Time-History of the Parameter Estimation Errors $e_{a}, e_{b}, e_{c}, e_{d}, e_{r}$

\section{Adaptive Controller Design for the Hybrid SynChronization DESIGN OF HYPERCHAOTIC YU SYSTEMS}

In this section, we design an adaptive controller for the hybrid synchronization of two identical hyperchaotic Yusystems (2012) with unknown parameters.

The hyperchaotic Yusystem is taken as the master system, whose dynamics is given by

$$
\begin{aligned}
& \dot{x}_{1}=\alpha\left(x_{2}-x_{1}\right) \\
& \dot{x}_{2}=\beta x_{1}-x_{1} x_{3}+\gamma x_{2}+x_{4} \\
& \dot{x}_{3}=e^{x_{1} x_{2}}-\delta x_{3} \\
& \dot{x}_{4}=-\varepsilon x_{1}
\end{aligned}
$$

where $\alpha, \beta, \gamma, \delta, \varepsilon$ are unknown parameters of the system and $x \in R^{4}$ is the state of the system. 
International Journal of Information Technology Convergence and Services (IJITCS) Vol.3, No.2, April 2013

The hyperchaotic Yu system is also taken as the slave system, whose dynamics is given by

$$
\begin{aligned}
& \dot{y}_{1}=\alpha\left(y_{2}-y_{1}\right)+u_{1} \\
& \dot{y}_{2}=\beta y_{1}-y_{1} y_{3}+\gamma y_{2}+y_{4}+u_{2} \\
& \dot{y}_{3}=e^{y_{1} y_{2}}-\delta y_{3}+u_{3} \\
& \dot{y}_{4}=-\varepsilon y_{1}+u_{4}
\end{aligned}
$$

Where $y \in R^{4}$ is the state and $u_{1}, u_{2}, u_{3}, u_{4}$ are the adaptivecontrollers to be designed using estimates $\hat{\alpha}(t), \hat{\beta}(t), \hat{\gamma}(t), \hat{\delta}(t), \hat{\varepsilon}(t)$ of the unknown parameters $\alpha, \beta, \gamma, \delta, \varepsilon$, respectively.

For the hybrid synchronization, the error $e$ is defined as

$$
\begin{aligned}
& e_{1}=y_{1}-x_{1} \\
& e_{2}=y_{2}+x_{2} \\
& e_{3}=y_{3}-x_{3} \\
& e_{4}=y_{4}+x_{4}
\end{aligned}
$$

A simple calculation gives the error dynamics

$$
\begin{aligned}
& \dot{e}_{1}=\alpha\left(y_{2}-x_{2}-e_{1}\right)+u_{1} \\
& \dot{e}_{2}=\beta\left(y_{1}+x_{1}\right)+\gamma e_{2}+e_{4}-y_{1} y_{3}-x_{1} x_{3}+u_{2} \\
& \dot{e}_{3}=-\delta e_{3}+e^{y_{1} y_{2}}-e^{x_{1} x_{2}}+u_{3} \\
& \dot{e}_{4}=-\varepsilon\left(y_{1}+x_{1}\right)+u_{4}
\end{aligned}
$$

Next, we choose a nonlinear controller for achieving hybrid synchronization as

$$
\begin{aligned}
& u_{1}=-\hat{\alpha}(t)\left(y_{2}-x_{2}-e_{1}\right)-k_{1} e_{1} \\
& u_{2}=-\hat{\beta}(t)\left(y_{1}+x_{1}\right)-\hat{\gamma}(t) e_{2}-e_{4}+y_{1} y_{3}+x_{1} x_{3}-k_{2} e_{2} \\
& u_{3}=\hat{\delta}(t) e_{3}-e^{y_{1} y_{2}}+e^{x_{1} x_{2}}-k_{3} e_{3} \\
& u_{4}=\hat{\varepsilon}(t)\left(y_{1}+x_{1}\right)-k_{4} e_{4}
\end{aligned}
$$

In Eq. (31), $k_{i},(i=1,2,3,4)$ are positive gains.

By the substitution of (31) into (30), the error dynamics is simplified as

$$
\begin{aligned}
& \dot{e}_{1}=(\alpha-\hat{\alpha}(t))\left(y_{2}-y_{1}\right)-k_{1} e_{1} \\
& \dot{e}_{2}=(\beta-\hat{\beta}(t))\left(y_{1}+x_{1}\right)+(\gamma-\hat{\gamma}(t)) e_{2}-k_{2} e_{2} \\
& \dot{e}_{3}=-(\delta-\hat{\delta}(t)) e_{3}-k_{3} e_{3} \\
& \dot{e}_{4}=-(\varepsilon-\hat{\varepsilon}(t))\left(y_{1}+x_{1}\right)-k_{4} e_{4}
\end{aligned}
$$


Next, we define the parameter estimation errors as

$$
\begin{aligned}
& e_{\alpha}(t)=\alpha-\hat{\alpha}(t) \\
& e_{\beta}(t)=\beta-\hat{\beta}(t) \\
& e_{\gamma}(t)=\gamma-\hat{\gamma}(t) \\
& e_{\delta}(t)=\delta-\hat{\delta}(t) \\
& e_{\varepsilon}(t)=\varepsilon-\hat{\varepsilon}(t)
\end{aligned}
$$

Differentiating (33) with respect to $t$, we get

$$
\dot{e}_{\alpha}(t)=-\dot{\hat{\alpha}}(t), \dot{e}_{\beta}(t)=-\dot{\hat{\beta}}(t), \dot{e}_{\gamma}(t)=-\dot{\hat{\gamma}}(t), \dot{e}_{\delta}(t)=-\dot{\hat{\delta}}(t), \quad \dot{e}_{\varepsilon}(t)=-\dot{\hat{\varepsilon}}(t)
$$

In view of (33), we can simplify the error dynamics (32) as

$$
\begin{aligned}
& \dot{e}_{1}=e_{\alpha}\left(y_{2}-x_{2}-e_{1}\right)-k_{1} e_{1} \\
& \dot{e}_{2}=e_{\beta}\left(y_{1}+x_{1}\right)+e_{\gamma} e_{2}-k_{2} e_{2} \\
& \dot{e}_{3}=-e_{\delta} e_{3}-k_{3} e_{3} \\
& \dot{e}_{4}=-e_{\varepsilon}\left(y_{1}+x_{1}\right)-k_{4} e_{4}
\end{aligned}
$$

We take the quadratic Lyapunov function

$$
V=\frac{1}{2}\left(e_{1}^{2}+e_{2}^{2}+e_{3}^{2}+e_{4}^{2}+e_{\alpha}^{2}+e_{\beta}^{2}+e_{\gamma}^{2}+e_{\delta}^{2}+e_{\varepsilon}^{2}\right),
$$

which is a positive definite function on $R^{9}$.

When we differentiate (35) along the trajectories of (32) and (33), we get

$$
\begin{aligned}
\dot{V}= & -k_{1} e_{1}^{2}-k_{2} e_{2}^{2}-k_{3} e_{3}^{2}-k_{4} e_{4}^{2}+e_{\alpha}\left[e_{1}\left(y_{2}-x_{2}-e_{1}\right)-\dot{\hat{\alpha}}\right]+e_{\beta}\left[e_{2}\left(y_{1}+x_{1}\right)-\dot{\hat{\beta}}\right] \\
& +e_{\gamma}\left[e_{2}^{2}-\dot{\hat{\gamma}}\right]+e_{\delta}\left[-e_{3}^{2}-\dot{\hat{\delta}}\right]+e_{\varepsilon}\left[-e_{4}\left(y_{1}+x_{1}\right)-\dot{\hat{\varepsilon}}\right]
\end{aligned}
$$

In view of Eq. (37), we take the parameter update law as

$$
\begin{array}{ll}
\dot{\hat{\alpha}}=e_{1}\left(y_{2}-x_{2}-e_{1}\right)+k_{5} e_{\alpha}, & \dot{\hat{\beta}}=e_{2}\left(y_{1}+x_{1}\right)+k_{6} e_{\beta}, \quad \dot{\hat{\gamma}}=e_{2}^{2}+k_{7} e_{\gamma} \\
\dot{\hat{\delta}}=-e_{3}^{2}+k_{8} e_{\delta}, & \dot{\hat{\varepsilon}}=-e_{4}\left(y_{1}+x_{1}\right)+k_{9} e_{\varepsilon}
\end{array}
$$

Theorem 5.1 The adaptive control law (31) along with the parameter update law (38), where $k_{i},(i=1,2, \ldots, 9)$ are positive gains, achieves global and exponential hybrid synchronization of the identical hyperchaotic Yu systems (27) and (28), where $\hat{\alpha}(t), \hat{\beta}(t), \hat{\gamma}(t), \hat{\delta}(t), \hat{\varepsilon}(t)$ are estimates of the unknown parameters $\alpha, \beta, \gamma, \delta, \varepsilon$, respectively. Moreover, all the parameter estimation errors converge to zero exponentially for all initial conditions. 
Proof.We prove the above result using Lyapunov stability theory [25].

Substituting the parameter update law (38) into (37), we get

$$
\dot{V}=-k_{1} e_{1}^{2}-k_{2} e_{2}^{2}-k_{3} e_{3}^{2}-k_{4} e_{4}^{2}-k_{5} e_{\alpha}^{2}-k_{6} e_{\beta}^{2}-k_{7} e_{\gamma}^{2}-k_{8} e_{\delta}^{2}-k_{9} e_{\varepsilon}^{2}
$$

which is a negative definite function on $R^{9}$.

This shows that the hybrid synchronization errors $e_{1}(t), e_{2}(t), e_{3}(t), e_{4}(t)$ and the parameter estimation errors $e_{\alpha}(t), e_{\beta}(t), e_{\gamma}(t), e_{\delta}(t), e_{\varepsilon}(t)$ are globally exponentially stable for all initial conditions. This completes the proof.

Next, we demonstrate our hybrid synchronization results with MATLAB simulations.

The classical fourth order Runge-Kutta method with time-step $h=10^{-8}$ has been applied to solve the hyperchaotic $\mathrm{Yu}$ systems (27) and (28) with the adaptive nonlinear controller(31) and the parameter update law (38). The feedback gains are taken as

$$
k_{i}=5,(i=1,2, \ldots, 9)
$$

The parameters of the hyperchaotic Yu systems are taken as in the hyperchaotic case, i.e.

$$
\alpha=10, \quad \beta=40, \quad \gamma=1, \delta=3, \varepsilon=8
$$

For simulations, the initial conditions of the hyperchaoticYu system (27) are chosen as

$$
x_{1}(0)=4, \quad x_{2}(0)=-2, \quad x_{3}(0)=8, \quad x_{4}(0)=-10
$$

Also, the initial conditions of the hyperchaotic Yusystem (28) are chosen as

$$
y_{1}(0)=16, \quad y_{2}(0)=8, \quad y_{3}(0)=12, \quad y_{4}(0)=-6
$$

Also, the initial conditions of the parameter estimates are chosen as

$$
\hat{\alpha}(0)=17, \quad \hat{\beta}(0)=-7, \quad \hat{\gamma}(0)=12, \quad \hat{\delta}(0)=-5, \quad \hat{\varepsilon}(0)=6
$$

Figure 6depicts the hybrid synchronization of the identical hyperchaoticYu systems.

Figure 7depicts the time-history of the hybrid synchronization errors $e_{1}, e_{2}, e_{3}, e_{4}$.

Figure 8depicts the time-history of the parameter estimation errors $e_{\alpha}, e_{\beta}, e_{\gamma}, e_{\delta}, e_{\varepsilon}$. 
International Journal of Information Technology Convergence and Services (IJITCS) Vol.3, No.2, April 2013
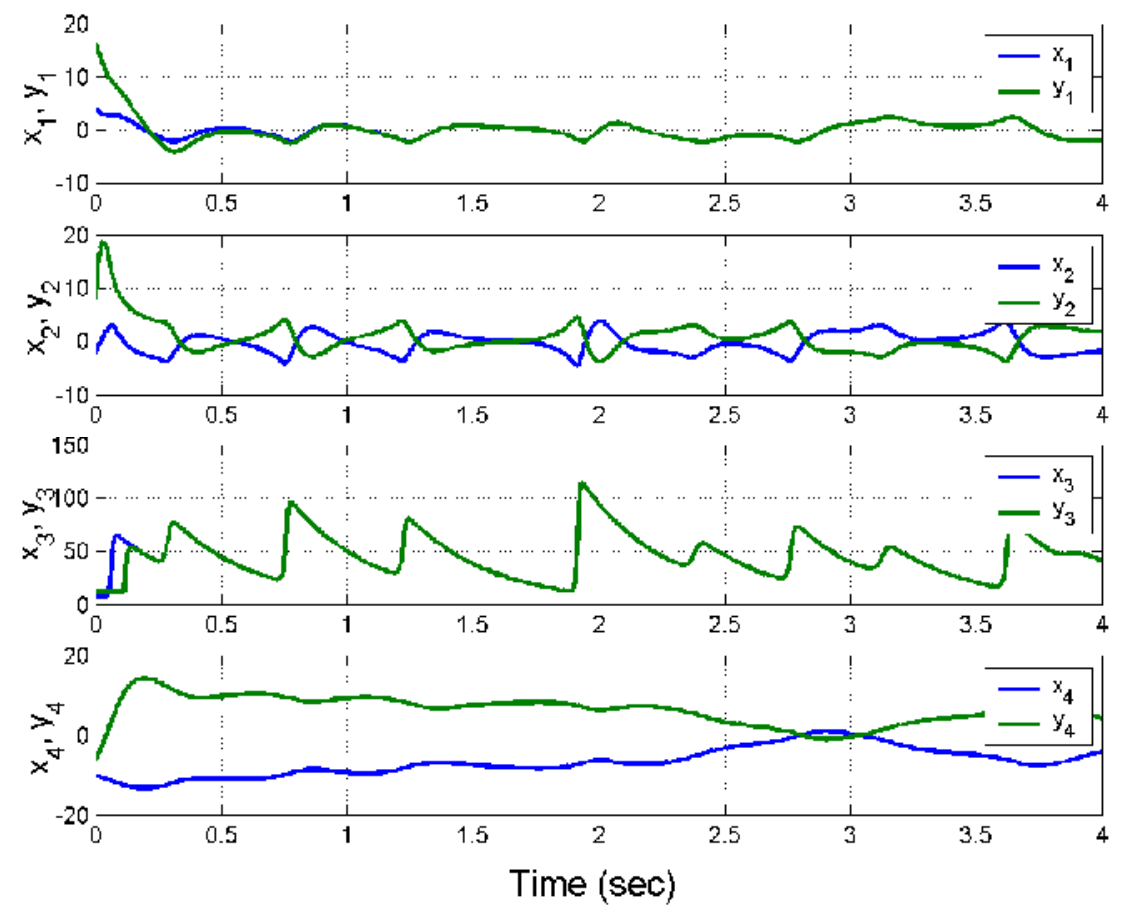

Figure 6.Hybrid Synchronization of Identical Hyperchaotic Yu Systems

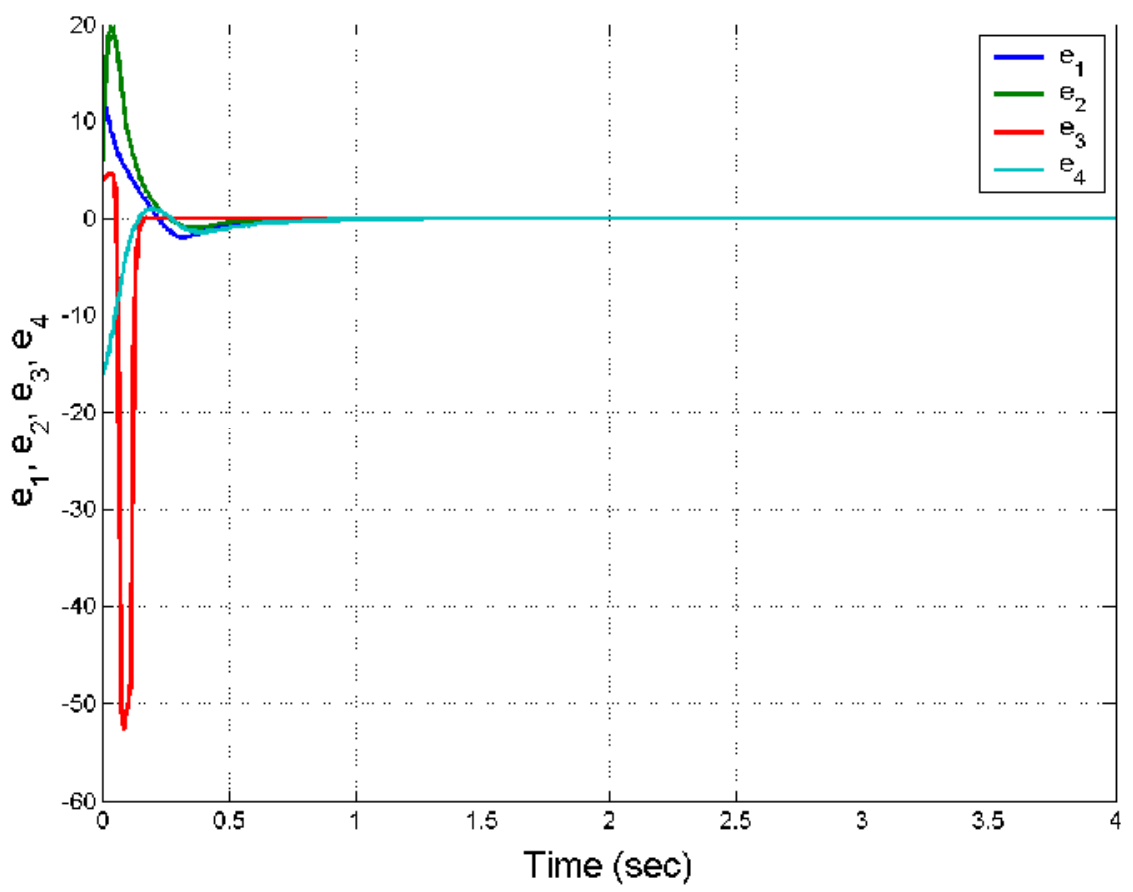

Figure 7. Time-History of the Hybrid Synchronization Errors $e_{1}, e_{2}, e_{3}, e_{4}$ 


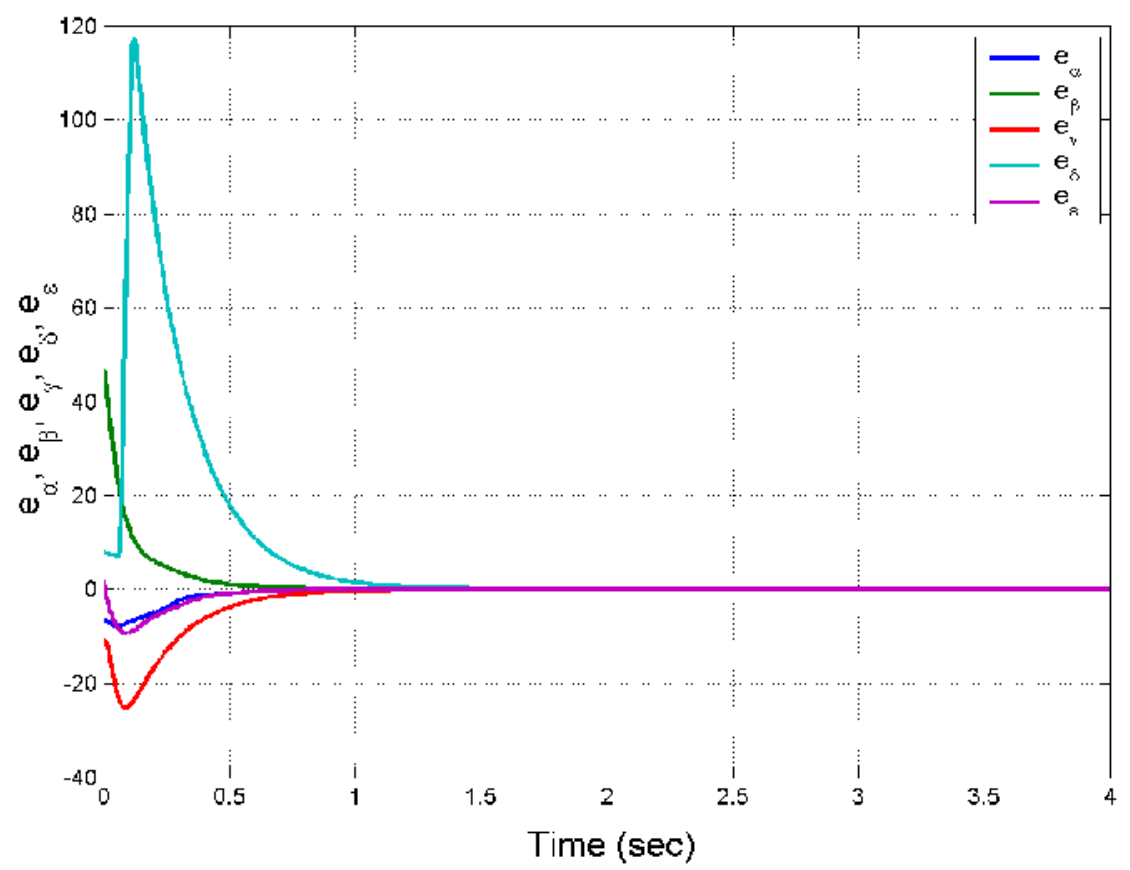

Figure 8. Time-History of the Parameter Estimation Errors $e_{\alpha}, e_{\beta}, e_{\gamma}, e_{\delta}, e_{\varepsilon}$

\section{AdaPtive Controller Design fOR the Hybrid SynChronization DESIGN OF HYPERCHAOTIC ZHENG AND HYPERCHAOTIC YU SYSTEMS}

In this section, we design an adaptive controller for the hybrid synchronization ofhyperchaoticZheng system (2010) and hyperchaotic Yusystem (2012) with unknown parameters.

The hyperchaotic Zhengsystem is taken as the master system, whose dynamics is given by

$$
\begin{aligned}
& \dot{x}_{1}=a\left(x_{2}-x_{1}\right)+x_{4} \\
& \dot{x}_{2}=b x_{1}+c x_{2}+x_{1} x_{3}+x_{4} \\
& \dot{x}_{3}=-x_{1}^{2}-r x_{3} \\
& \dot{x}_{4}=-d x_{2}
\end{aligned}
$$

where $a, b, c, d, r$ are unknown parameters of the system.

The hyperchaotic $\mathrm{Yu}$ system is also taken as the slave system, whose dynamics is given by

$$
\begin{aligned}
& \dot{y}_{1}=\alpha\left(y_{2}-y_{1}\right)+u_{1} \\
& \dot{y}_{2}=\beta y_{1}-y_{1} y_{3}+\gamma y_{2}+y_{4}+u_{2} \\
& \dot{y}_{3}=e^{y_{1} y_{2}}-\delta y_{3}+u_{3} \\
& \dot{y}_{4}=-\varepsilon y_{1}+u_{4}
\end{aligned}
$$

where $\alpha, \beta, \gamma, \delta, \varepsilon$ are unknown parametersand $u_{1}, u_{2}, u_{3}, u_{4}$ are the adaptivecontrollers. 
International Journal of Information Technology Convergence and Services (IJITCS) Vol.3, No.2, April 2013

For the hybrid synchronization, the error $e$ is defined as

$$
e_{1}=y_{1}-x_{1}, e_{2}=y_{2}+x_{2}, e_{3}=y_{3}-x_{3}, e_{4}=y_{4}+x_{4}
$$

A simple calculation gives the error dynamics

$$
\begin{aligned}
& \dot{e}_{1}=\alpha\left(y_{2}-y_{1}\right)-a\left(x_{2}-x_{1}\right)-x_{4}+u_{1} \\
& \dot{e}_{2}=\beta y_{1}+\gamma y_{2}+e_{4}+b x_{1}+c x_{2}-y_{1} y_{3}+x_{1} x_{3}+u_{2} \\
& \dot{e}_{3}=-\delta y_{3}+r x_{3}+e^{y_{1} y_{2}}+x_{1}^{2}+u_{3} \\
& \dot{e}_{4}=-\varepsilon y_{1}-d x_{2}+u_{4}
\end{aligned}
$$

Next, we choose a nonlinear controller for achieving hybrid synchronization as

$$
\begin{aligned}
& u_{1}=-\hat{\alpha}(t)\left(y_{2}-y_{1}\right)+\hat{a}(t)\left(x_{2}-x_{1}\right)+x_{4}-k_{1} e_{1} \\
& u_{2}=-\hat{\beta}(t) y_{1}-\hat{\gamma}(t) y_{2}-e_{4}-\hat{b}(t) x_{1}-\hat{c}(t) x_{2}+y_{1} y_{3}-x_{1} x_{3}-k_{2} e_{2} \\
& u_{3}=\hat{\delta}(t) y_{3}-\hat{r}(t) x_{3}-e^{y_{1} y_{2}}-x_{1}^{2}-k_{3} e_{3} \\
& u_{4}=\hat{\varepsilon}(t) y_{1}+\hat{d}(t) x_{2}-k_{4} e_{4}
\end{aligned}
$$

where $k_{i},(i=1,2,3,4)$ are positive gains.

By the substitution of (44) into (43), the error dynamics is obtained as

$$
\begin{aligned}
& \dot{e}_{1}=(\alpha-\hat{\alpha}(t))\left(y_{2}-y_{1}\right)-(a-\hat{a}(t))\left(x_{2}-x_{1}\right)-k_{1} e_{1} \\
& \dot{e}_{2}=(\beta-\hat{\beta}(t)) y_{1}+(\gamma-\hat{\gamma}(t)) y_{2}+(b-\hat{b}(t)) x_{1}+(c-\hat{c}(t)) x_{2}-k_{2} e_{2} \\
& \dot{e}_{3}=-(\delta-\hat{\delta}(t)) y_{3}+(r-\hat{r}(t)) x_{3}-k_{3} e_{3} \\
& \dot{e}_{4}=-(\varepsilon-\hat{\varepsilon}(t)) y_{1}-(d-\hat{d}(t)) x_{2}-k_{4} e_{4}
\end{aligned}
$$

Next, we define the parameter estimation errors as

$$
\begin{aligned}
& e_{a}(t)=a-\hat{a}(t), \quad e_{b}(t)=b-\hat{b}(t), \quad e_{c}(t)=c-\hat{c}(t), e_{d}(t)=d-\hat{d}(t) \\
& e_{r}(t)=r-\hat{r}(t), \quad e_{\alpha}(t)=\alpha-\hat{\alpha}(t), e_{\beta}(t)=\beta-\hat{\beta}(t), e_{\gamma}(t)=\gamma-\hat{\gamma}(t) \\
& e_{\delta}(t)=\delta-\hat{\delta}(t), e_{\varepsilon}(t)=\varepsilon-\hat{\varepsilon}(t)
\end{aligned}
$$

Differentiating (46) with respect to $t$, we get

$$
\begin{aligned}
& \dot{e}_{a}(t)=-\dot{\hat{a}}(t), \dot{e}_{b}(t)=-\dot{\hat{b}}(t), \dot{e}_{c}(t)=-\dot{\hat{c}}(t), \dot{e}_{d}(t)=-\dot{\hat{d}}(t), \dot{e}_{r}(t)=-\dot{\hat{r}}(t) \\
& \dot{e}_{\alpha}(t)=-\dot{\hat{\alpha}}(t), \dot{e}_{\beta}(t)=-\dot{\hat{\beta}}(t), \dot{e}_{\gamma}(t)=-\dot{\hat{\gamma}}(t), \dot{e}_{\delta}(t)=-\dot{\hat{\delta}}(t), \dot{e}_{\varepsilon}(t)=-\dot{\hat{\varepsilon}}(t)
\end{aligned}
$$


International Journal of Information Technology Convergence and Services (IJITCS) Vol.3, No.2, April 2013

In view of (46), we can simplify the error dynamics (45) as

$$
\begin{aligned}
& \dot{e}_{1}=e_{\alpha}\left(y_{2}-y_{1}\right)-e_{a}\left(x_{2}-x_{1}\right)-k_{1} e_{1} \\
& \dot{e}_{2}=e_{\beta} y_{1}+e_{\gamma} y_{2}+e_{b} x_{1}+e_{c} x_{2}-k_{2} e_{2} \\
& \dot{e}_{3}=-e_{\delta} y_{3}+e_{r} x_{3}-k_{3} e_{3} \\
& \dot{e}_{4}=-e_{\varepsilon} y_{1}-e_{d} x_{2}-k_{4} e_{4}
\end{aligned}
$$

We take the quadratic Lyapunov function

$$
V=\frac{1}{2}\left(e_{1}^{2}+e_{2}^{2}+e_{3}^{2}+e_{4}^{2}+e_{a}^{2}+e_{b}^{2}+e_{c}^{2}+e_{d}^{2}+e_{r}^{2}+e_{\alpha}^{2}+e_{\beta}^{2}+e_{\gamma}^{2}+e_{\delta}^{2}+e_{\varepsilon}^{2}\right)
$$

When we differentiate (48) along the trajectories of (45) and (46), we get

$$
\begin{aligned}
\dot{V}= & -k_{1} e_{1}^{2}-k_{2} e_{2}^{2}-k_{3} e_{3}^{2}-k_{4} e_{4}^{2}+e_{a}\left[-e_{1}\left(x_{2}-x_{1}\right)-\dot{\hat{a}}\right]+e_{b}\left[e_{2} x_{1}-\dot{\hat{b}}\right]+e_{c}\left[e_{2} x_{2}-\dot{\hat{c}}\right] \\
& +e_{d}\left[-e_{4} x_{2}-\dot{\hat{d}}\right]+e_{r}\left[e_{3} x_{3}-\dot{\hat{r}}\right]+e_{\alpha}\left[e_{1}\left(y_{2}-y_{1}\right)-\dot{\hat{\alpha}}\right]+e_{\beta}\left[e_{2} y_{1}-\dot{\hat{\beta}}\right] \\
& +e_{\gamma}\left[e_{2} y_{2}-\dot{\hat{\gamma}}\right]+e_{\delta}\left[-e_{3} y_{3}-\dot{\hat{\delta}}\right]+e_{\varepsilon}\left[-e_{4} y_{1}-\dot{\hat{\varepsilon}}\right]
\end{aligned}
$$

In view of Eq. (50), we take the parameter update law as

$$
\begin{array}{lll}
\dot{\hat{a}}=-e_{1}\left(x_{2}-x_{1}\right)+k_{5} e_{a}, & \dot{\hat{b}}=e_{2} x_{1}+k_{6} e_{b}, & \dot{\hat{c}}=e_{2} x_{2}+k_{7} e_{c} \\
\dot{\hat{d}}=-e_{4} x_{2}+k_{8} e_{d}, & \dot{\hat{r}}=e_{3} x_{3}+k_{9} e_{r}, & \dot{\hat{\alpha}}=e_{1}\left(y_{2}-y_{1}\right)+k_{10} e_{\alpha} \\
\dot{\hat{\beta}}=e_{2} y_{1}+k_{11} e_{\beta}, & \dot{\hat{\gamma}}=e_{2} y_{2}+k_{12} e_{\gamma}, & \dot{\hat{\delta}}=-e_{3} y_{3}+k_{13} e_{\delta} \\
\dot{\hat{\varepsilon}}=-e_{4} y_{1}+k_{14} e_{\varepsilon} &
\end{array}
$$

Theorem 6.1 The adaptive control law (44) along with the parameter update law (51), where $k_{i},(i=1,2, \ldots, 14)$ are positive gains, achieves global and exponential hybrid synchronization of the hyperchaotic Zheng system (40) hyperchaotic Yu system (41), where $\hat{a}(t), \hat{b}(t), \hat{c}(t), \hat{d}(t)$, $\hat{r}(t), \hat{\alpha}(t), \hat{\beta}(t), \hat{\gamma}(t), \hat{\delta}(t), \hat{\varepsilon}(t)$ are estimates of the unknown parameters $a, b, c, d, r$, $\alpha, \beta, \gamma, \delta, \varepsilon$, respectively. Moreover, all the parameter estimation errors converge to zero exponentially for all initial conditions.

Proof.We prove the above result using Lyapunov stability theory [25].Substituting the parameter update law (51) into (50), we get

$$
\begin{aligned}
\dot{V}= & -k_{1} e_{1}^{2}-k_{2} e_{2}^{2}-k_{3} e_{3}^{2}-k_{4} e_{4}^{2}-k_{5} e_{a}^{2}-k_{6} e_{b}^{2}-k_{7} e_{c}^{2}-k_{8} e_{d}^{2}-k_{9} e_{r}^{2} \\
& -k_{10} e_{\alpha}^{2}-k_{11} e_{\beta}^{2}-k_{12} e_{\gamma}^{2}-k_{13} e_{\delta}^{2}-k_{14} e_{\varepsilon}^{2}
\end{aligned}
$$

which is a negative definite function on $R^{14}$. 
This shows that the hybrid synchronization errors $e_{1}(t), e_{2}(t), e_{3}(t), e_{4}(t)$ and the parameter estimation errors $e_{a}(t), e_{b}(t), e_{c}(t), e_{d}(t), e_{r}(t), e_{\alpha}(t), e_{\beta}(t), e_{\gamma}(t), e_{\delta}(t), e_{\varepsilon}(t)$ are globally exponentially stable for all initial conditions. This completes the proof.

For simulations, theclassical fourth order Runge-Kutta method with time-step $h=10^{-8}$ has been applied to solve the hyperchaotic Li systems (27) and (28) with the adaptive nonlinear controller(31) and the parameter update law (38). The feedback gains are taken as $k_{i}=5,(i=1,2, \ldots, 14)$. The parameters of the hyperchaotic Zheng and hyperchaotic Yu systems are takenas $a=20, b=14, c=10.6, \quad d=4, r=2.8, \alpha=10, \beta=40, \gamma=1, \delta=3$ and $\varepsilon=8$.

For simulations, the initial conditions of the hyperchaotic Zheng system (40) are chosen as

$$
x_{1}(0)=4, \quad x_{2}(0)=9, \quad x_{3}(0)=1, \quad x_{4}(0)=4
$$

Also, the initial conditions of the hyperchaotic Yu system (41) are chosen as

$$
y_{1}(0)=8, \quad y_{2}(0)=3, \quad y_{3}(0)=1, \quad y_{4}(0)=2
$$

Also, the initial conditions of the parameter estimates are chosen as

$$
\hat{a}(0)=2, \hat{b}(0)=6, \hat{c}(0)=3, \hat{d}(0)=-3, \hat{r}(0)=-1, \hat{\alpha}(0)=7, \hat{\beta}(0)=4, \hat{\gamma}(0)=9, \hat{\delta}(0)=5, \hat{\varepsilon}(0)=4
$$

Figure 9depicts the hybrid synchronization of hyperchaoticZheng and hyperchaotic Yu systems. Figure 10depicts the time-history of the hybrid synchronization errors $e_{1}, e_{2}, e_{3}, e_{4}$. Figure 11 depicts the time-history of the parameter estimation errors $e_{a}, e_{b}, e_{c}, e_{d}, e_{r}$. Figure 12depicts the time-history of the parameter estimation errors $e_{\alpha}, e_{\beta}, e_{\gamma}, e_{\delta}, e_{\varepsilon}$.
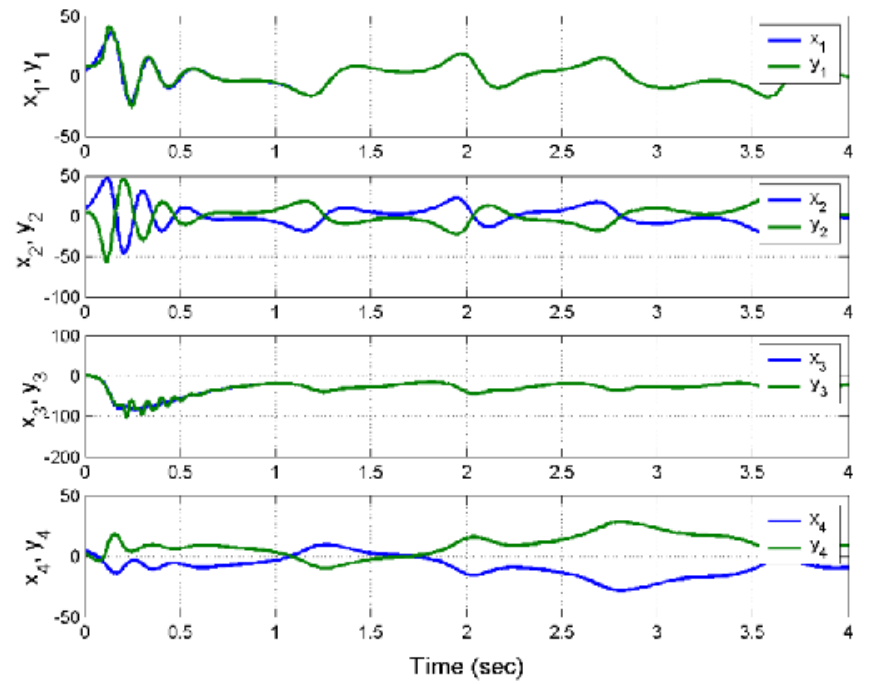

Figure 9.Hybrid Synchronization of Hyperchaotic Xu and Lu Systems 
International Journal of Information Technology Convergence and Services (IJITCS) Vol.3, No.2, April 2013

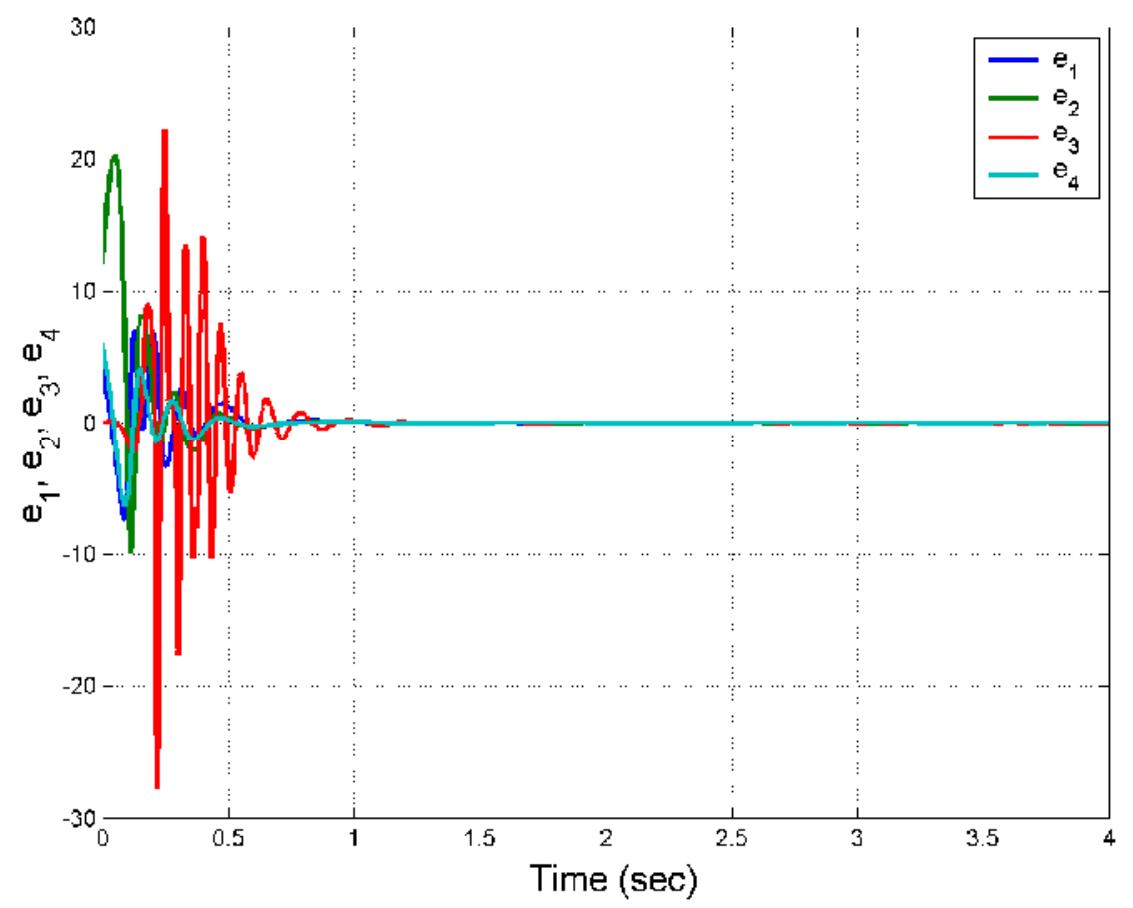

Figure 10. Time-History of the Hybrid Synchronization Errors $e_{1}, e_{2}, e_{3}, e_{4}$

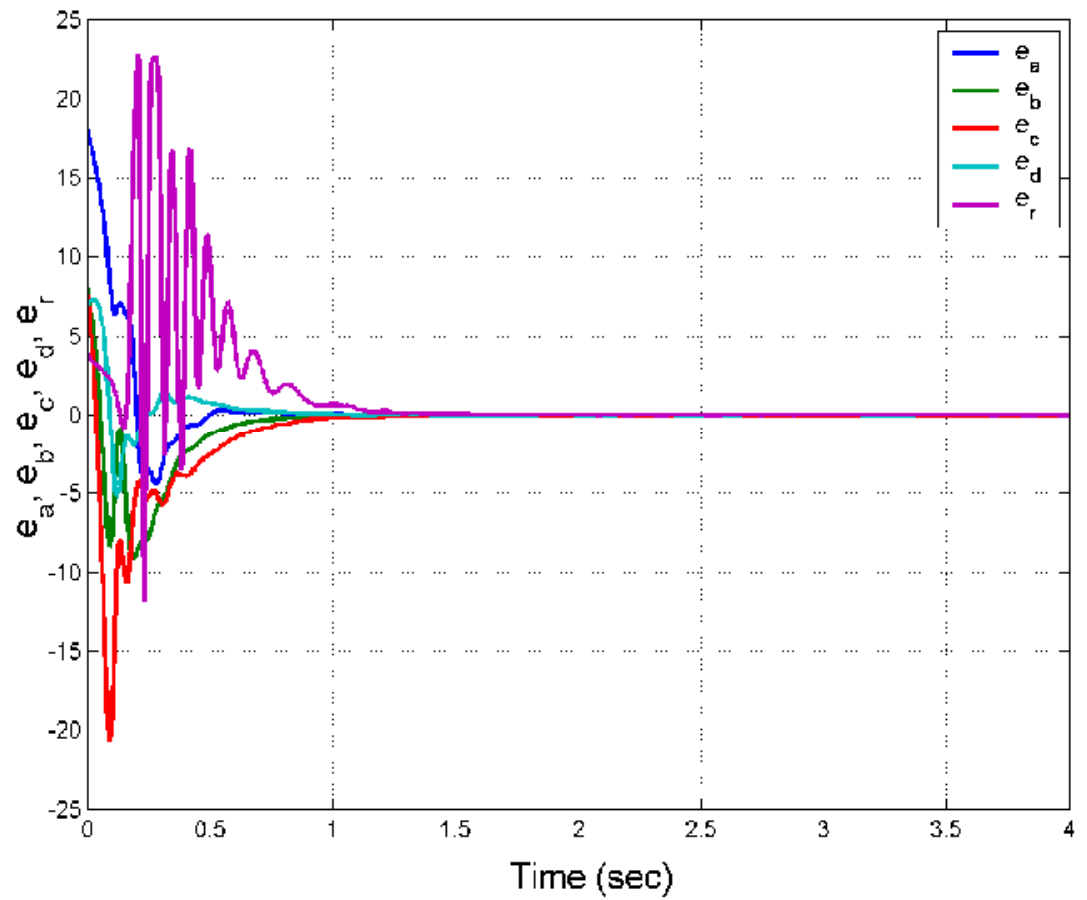

Figure 11. Time-History of the Parameter Estimation Errors $e_{a}, e_{b}, e_{c}, e_{d}$ 


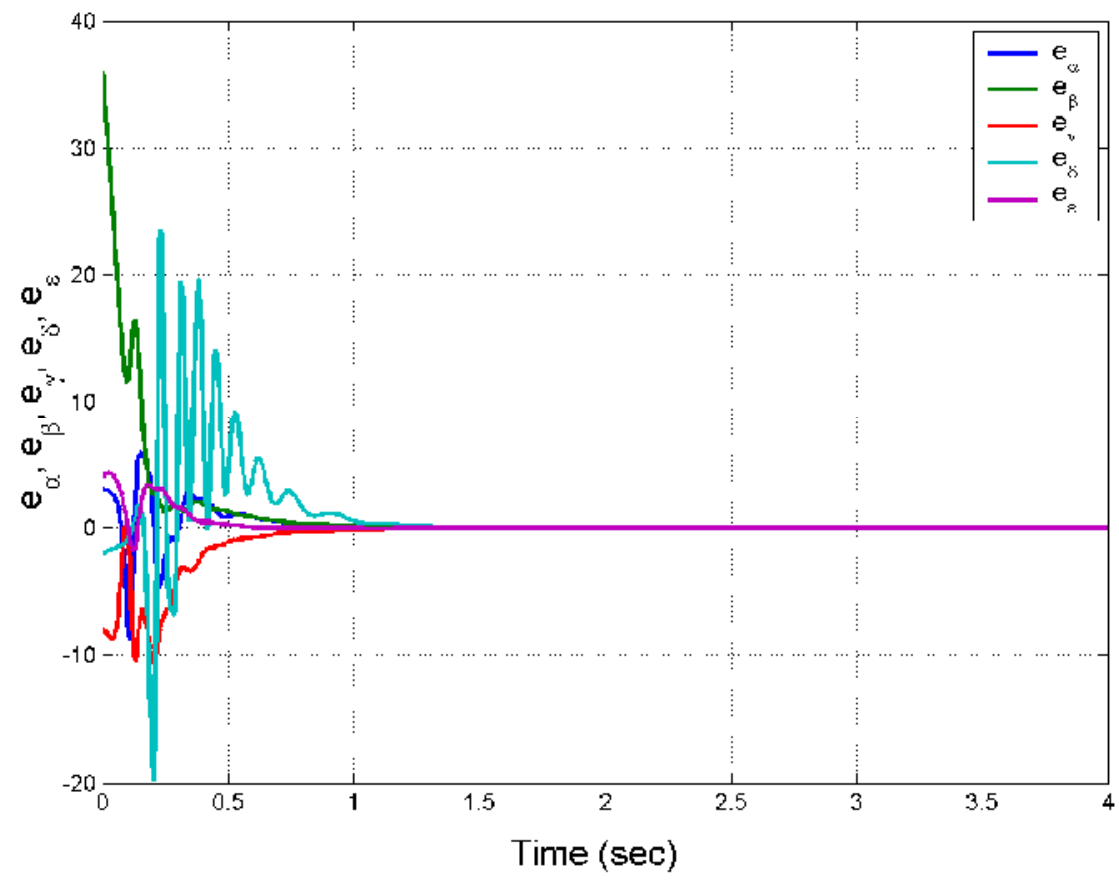

Figure 12. Time-History of the Parameter Estimation Errors $e_{\alpha}, e_{\beta}, e_{\gamma}, e_{\delta}, e_{\varepsilon}$

\section{Conclusions}

This paper derived new results for the active synchronizer design for achieving hybrid synchronization of hyperchaoticZhengsystems (2010) and hyperchaotic Yu systems (2012). Using Lyapunov control theory,adaptive control laws were derived for globally hybrid synchronizing the states of identical hyperchaotic Zheng systems, identical hyperchaotic Yu systems and non-identical hyperchaotic Zheng and Yu systems. Numerical simulations using MATLABwere shown to validate and illustrate the hybrid synchronization results for hyperchaotic Zheng and Yu systems.

\section{REFERENCES}

[1] Rössler, O.E. (1979) “An equation for hyperchaos,” Physics Letters A, Vol. 71, pp 155-157.

[2] Huang, Y. \& Yang, X.S. (2006) "Hyperchaos and bifurcation in a new class of four-dimensional Hopfield neural networks," Neurocomputing, Vol. 69, pp 13-15.

[3] Machado, L.G., Savi, M.A. \& Pacheco, P.M.C.L. (2003) "Nonlinear dynamics and chaos in coupled shape memory oscillators," International Journal of Solids and Structures, Vol. 40, No. 19, pp. 51395156.

[4] Tao, Y. (1999) "Chaotic secure communication systems - history and new results", Telecommun. Review, Vol. 9, pp 597-634.

[5] Li, C., Liao, X. \& Wong, K.W. (2005) "Lag synchronization of hyperchaos with applications to secure communications," Chaos, Solitons \& Fractals, Vol. 23, No. 1, pp 183-193.

[6] Prokhorov, M.D. \& Ponomarenko, V.I. (2008) "Encryption and decryption of information in chaotic communication systems governed by delay-differential equations," Chaos, Solitons \& Fractals, Vol. 35, No. 5, pp 871-877.

[7] Yassen, M.T. (2008) "Synchronization hyperchaos of hyperchaotic systems", Chaos, Solitons and Fractals, Vol. 37, pp 465-475. 
[8] Ott, E., Grebogi, C. \& Yorke, J.A. (1990) “Controlling chaos”, Phys. Rev. Lett., Vol. 64, pp 11961199.

[9] Pecora, L.M. \& Carroll, T.L. (1990) "Synchronization in chaotic systems", Phys. Rev. Lett., Vol. 64, pp 821-824.

[10] Bowong, S. \&Kakmeni, F.M.M. (2004) "Synchronization of uncertain chaotic systems via backstepping approach," Chaos, Solitons \& Fractals, Vol. 21, No. 4, pp 999-1011.

[11] Suresh, R, \& Sundarapandian, V. (2012) "Global chaos synchronization of WINDMI and Coullet chaotic systems by backstepping control”, Far East J. Math. Sciences, Vol. 67, No. 2, pp 265-287.

[12] Suresh, R. \& Sundarapandian, V. (2012) "Hybrid synchronization of n-scroll Chua and Lur'e chaotic systems via backstepping control with novel feedback", Arch. Control Sciences, Vol. 22, No. 3, pp 255-278.

[13] Senejohnny, D.M. \& Delavari, H. (2012) "Active sliding observer scheme based fractional chaos synchronization,” Comm. Nonlinear Sci. Numerical Simulation, Vol. 17, No. 11, pp 4373-4383.

[14] Sundarapandian, V. (2012) "Anti-synchronization of hyperchaotic Xu systems via sliding mode control”, International Journal of Embedded Systems, Vol. 2, No. 2, pp 51-61.

[15] Sundarapandian, V. (2013) "Anti-synchronizing sliding controller design for identical Pan systems," International Journal of Computational Science and Information Technology, Vol. 1, No. 1, pp 1-9.

[16] Huang, L. Feng, R. \& Wang, M. (2004) "Synchronization of chaotic systems via nonlinear control," Physics Letters A, Vol. 320, No. 4, pp 271-275.

[17] Lei, Y., Xu, W. \& Zheng, H. (2005) "Synchronization of two chaotic nonlinear gyros using active control," Physics Letters A, Vol. 343, pp 153-158.

[18] Sundarapandian, V. (2012) "Adaptive control and synchronization of a generalized Lotka-Volterra system," International Journal of Bioinformatics and Biosciences, Vol. 1, No. 1, pp 1-12.

[19] Sundarapandian, V. (2013) “Adaptive controller and synchronizer design for hyperchaotic Zhou system with unknown parameters,'International Journal of Information Technology, Modeling and Computing, Vol. 1, No. 1, pp 18-32.

[20] Zhao, J. \& Lü, J. (2006) "Using sampled-data feedback control and linear feedback synchronization in a new hyperchaotic system," Chaos, Solitons \& Fractals, Vol. 35, pp. 376-382.

[21] Ma, H., Deshmukh, V., Butcher, E. \& Averina, V. (2005) "Delayed state feedback and chaos control for time-periodic systems via a symbolic approach", Communications in Nonlinear Science and Numerical Simulation, Vol. 10, No. 5, pp 479-497.

[22] Zheng, S., Dong, G. \& Bi, Q. (2010) “A new hyperchaotic system and its synchronization,” Applied Mathematics and Computation, Vol. 215, pp 3192-3200.

[23] Yu, F., Wang, C.H., Hu, Y. \& Yin, J.W. (2012) "Anti-synchronization of a novel hyperchaotic system with parameter mismatch and external disturbances," Pramana-Journal of Physics, Vol. 79, No. 1, pp 81-93.

[24] Sastry, S. \& Bodson, M. (2011) Adaptive Control: Stability, Convergence and Robustness, Dover.

[25] Hahn, W. (1967) The Stability of Motion, Springer, Berlin. 